\title{
Balancing-Related Model Reduction for Parabolic Control Systems
}

\author{
Peter Benner* \\ * Max Planck Institute for Dynamics of Complex Technical Systems, \\ Magdeburg, Germany (e-mail: benner@mpi-magdeburg.mpg.de).
}

\begin{abstract}
Balanced truncation (BT) is a well established model reduction technique for linear ordinary differential equations. If the original dynamics are described by an instationary PDE, then BT is usually applied to the large-scale linear system resulting from a spatial semidiscretization using finite elements/volumes/differences. We will discuss this approach as well as a variant of BT based on balancing the solution of the linear-quadratic Gaussian (LQG) algebraic Riccati equations, called LQG BT, allowing the reduction of unstable systems and yielding a stabilizing feedback controller as a by-product. The error between reduced-order model and original system is split into discretization and model reduction components. We discuss the resulting error bounds and ways how to exploit this in order to adaptively choose the reduced system order. Numerical examples support our findings.
\end{abstract}

Keywords: Model order reduction; balanced truncation; LQG balancing; Lyapunov equation; algebraic Riccati equation.

\section{INTRODUCTION}

We consider control problems for parabolic partial differential equations of the form

$$
\partial_{t} \mathbf{x}-\nabla(a(\xi) \cdot \nabla \mathbf{x})+b(\xi) \cdot \nabla \mathbf{x}+c(\xi) \mathbf{x}=B_{p c}(\xi) u(t),
$$

with $(t, \xi) \in Q:=[0, T] \times \Omega, \Omega \subset \mathbb{R}^{d}$, together with initial and boundary conditions

$$
\begin{aligned}
\alpha(\xi) \mathbf{x}+\beta(\xi) \partial_{\eta} \mathbf{x} & =B_{b c}(\xi) u(t), & & \xi \in \partial \Omega, t \in[0, T] \\
\mathbf{x}(0, \xi) & =\mathbf{x}_{0}(\xi), & & \xi \in \Omega,
\end{aligned}
$$

We call (1)-(2) a parabolic control system. Examples include the control and optimization of instationary heat equations, instationary convection-diffusion equations, and linear reaction-diffusion equations. Here, $\mathbf{x}(t, \xi)$ represents the state of the system, while $u(t) \in \mathbb{R}^{m}$ denotes controls or more general, input parameters. We assume throughout that $u \in L_{2}\left(0, T ; \mathbb{R}^{m}\right)$. We allow $T=\infty$. Furthermore, we assume that for fixed $t \in(0, T), \mathbf{x}(t) \in \mathcal{V}$, where $\mathcal{V}$ is a dense subspace of the state space $\mathcal{X}$ which is assumed to be a Hilbert space $\left(\mathcal{X}=L_{2}(\Omega)\right.$ for standard parabolic control systems). For analysis and numerical solution, we assume that $\mathcal{V}$ and $\mathcal{X}$ induce a Gelfand triple $\mathcal{V} \subset \mathcal{X} \subset \mathcal{V}^{\prime}$, where $\mathcal{V}^{\prime}$ is the dual space of $\mathcal{V}$. In general, we understand $(2)$ in a weak sense so that solutions satisfy $\mathbf{x} \in L_{2}(0, T ; \mathcal{V})$ and $\partial_{t} \mathbf{x} \in L_{2}(0, T ; \mathcal{X})$. The subspace $\mathcal{V}$ will vary depending on the boundary conditions and geometry of $\Omega$; a classical example for homogeneous Dirichlet boundaries $(\beta \equiv 0)$ and point control $\left(B_{b c}=0\right.$; if $B_{p c}=0$, we speak of a boundary control problem) is $\mathcal{V}=H_{0}^{1}(\Omega)$, see, e.g., Banks and Kunisch (1984); Rosen and Wang (1995), where this particular setting is detailed. Furthermore, throughout we assume the coefficient functions in (1)-(2) are such that a unique weak solution exists for any feasible input function $u$ and any $\mathbf{x}_{0} \in \mathcal{X}$. For a comprehensive treatment of control problems in this and more abstract settings see, e.g., Bensoussan et al. (2007);
Curtain and Zwart (1995); Lasiecka and Triggiani (2000); Tröltzsch (2010).

In system and control theory, usually (1)-(2) is combined with an output equation

$$
\mathbf{y}(t)=C(\xi) \mathbf{x}(t, \xi), \quad \xi \in \Omega, \quad t \in[0, T],
$$

where $C(\xi): \mathcal{X} \rightarrow \mathbb{R}^{p}$ is a linear operator. This equation takes into account that in practise, usually only limited information about the state is accessible for control design. If, for example, $\mathbf{x}$ is continuous in $\bar{\Omega}$, then $C(\xi)$ might just pick $p$ values of the state at the positions $\xi_{1}, \ldots, \xi_{p}$. We will make use of (3) throughout this paper. On the other hand, we will not take into account control or state constraints which are usually present in optimization problems (see, e.g., Tröltzsch (2010)) as these will not influence the model reduction procedure. But note that the model reduction approach discussed here can be very helpful when solving control- or state-constrained optimization problems with standard quadratic or nonlinear programming techniques as the dimension of the mathematical programs resulting from a full discretization of the PDE constrained optimization might be way too large to be treatable with standard optimization software.

When solving parabolic control problems numerically, one usual approach is to first semi-discretize (1)-(3) in space, using, e.g., finite difference or finite element methods, and then to apply modern control synthesis techniques like linear-quadratic regulator/Gaussian design or robust control techniques like $\mathrm{H}_{2}-\mathrm{H}_{\infty}$-control, see, e.g., Banks and Kunisch (1984); Benner (2004); Burns and Hulsing (2001); Morris (1996); Morris and Navasca (2005). A major problem posed by these approaches is that the resulting controller is a finite-dimensional control system of at least the order of the semi-discretized plant model. For 2- and 3D problems, this ranges from several thousands to millions 
of state-space variables - a controller order that can not be implemented in control engineering practice. Moreover, using classical computational methods to compute such a controller might pose its own challenge despite the fact that there has been significant progress in solving largescale control problems, see, e.g., Benner (2004); Morris and Navasca (2005).

One remedy for all the abovementioned problems is model reduction. The idea is to replace the infinite-dimensional parabolic control system (1)-(3) by a finite-dimensional linear time-invariant system

$$
\begin{aligned}
\dot{\hat{x}}(t) & =\hat{A} \hat{x}(t)+\hat{B} u(t), & t>0, & \hat{x}(0)=\hat{x}^{0}, \\
\hat{y}(t) & =\hat{C} \hat{x}(t), & t \geq 0, &
\end{aligned}
$$

of order $r$, i.e., $\hat{x}(t) \in \mathbb{R}^{r}$, and matrices $\hat{A}, \hat{B}, \hat{C}$ of appropriate sizes, so that

(1) the same inputs $u \in L_{2}\left(0, T ; \mathbb{R}^{m}\right)$ used in (1)-(2) can be applied to (4), and

(2) the outputs produced by (1)-(3) and (4) are "close" to each other for all admissible input functions $u$ ideally, they satisfy

$$
\|y-\hat{y}\|_{L_{2}\left(0, T ; \mathbb{R}^{m}\right)} \leq \tau\|u\|_{L_{2}\left(0, T ; \mathbb{R}^{p}\right)}
$$

for a given error tolerance $\tau>0$.

(In the following, $L_{2}$-norms will be simply denoted by $\|$. $\|$ - the concrete space will be clear from the context.)

We will consider system-theoretic methods for model reduction of stable and unstable systems based on balancing. (See Antoulas (2005); Benner et al. (2005); Obinata and Anderson (2001) for introductions to these methods.) These approaches have the advantage of providing error bounds that are helpful in achieving (5). One model reduction approach often used in PDE-constrained optimization is proper orthogonal decomposition (POD), also known as Karhunen-Loève decomposition, see, e.g., Hinze and Volkwein (2005); Kunisch and Volkwein (2001). For linear PDE problems this approach turns out to be just an approximation to balanced truncation (Willcox and Peraire (2002)) and is thus inferior with respect to accuracy. As we will see, the computational complexity of balanced truncation is similar to that of POD so that balanced truncation appears to be preferable for model reduction of linear PDE control and optimisation problems.

The remainder of the paper is organised as follows. In Section 2, we will discuss some theoretical results from Curtain (2003); Glover et al. (1988) regarding balancingrelated model reduction for infinite-dimensional systems. These are applicable to parabolic control systems under certain assumptions. Unfortunately, computationally the suggested techniques of computing the reduced-order model directly from the infinite-dimensional system are not feasible in general. Thus, we discuss the combination of spatial semi-discretization (by finite element/differences methods) and finite-dimensional balanced truncation in Section 3. Here we employ techniques discussed for largescale, finite-dimensional systems as discussed, e.g., in Benner (2004, 2009); Benner et al. (2008). The computational efficiency is demonstrated by numerical examples in Section 4. Conclusions and outlook are given in Section 5.

\section{ABSTRACT BALANCED TRUNCATION}

For the abstract setting, we will assume a linear timeinvariant system in the following form:

$$
\Sigma:\left\{\begin{array}{l}
\dot{\mathbf{x}}=\mathbf{A x}+\mathbf{B u}, \quad \mathbf{x}(0)=\mathbf{x}_{0} \in \mathcal{X}, \\
\mathbf{y}=\mathbf{C x},
\end{array}\right.
$$

with linear operators

$$
\mathbf{A}: \operatorname{dom}(\mathbf{A}) \subset \mathcal{X} \rightarrow \mathcal{X}, \quad \mathbf{B}: \mathcal{U} \rightarrow \mathcal{X}, \quad \mathbf{C}: \mathcal{X} \rightarrow \mathcal{Y},
$$

where the state space $\mathcal{X}$, the control space $\mathcal{U}$ and the observation or output space $\mathcal{Y}$ are assumed to be Hilbert spaces. The parabolic control system (1)-(3) defined in the Introduction is an example for this setting, where we have used $\mathcal{U} \equiv \mathbb{R}^{m}$ in (1) and $\mathcal{Y} \equiv \mathbb{R}^{p}$ in (3). Hence we use $u, y$ rather than $\mathbf{u}, \mathbf{y}$ there to indicate that our inputs and outputs are not distributed functions in this setting. The latter assumptions will be used mostly in the applications considered later.

We will employ the following assumptions, where we refer to Curtain and Zwart (1995); Glover et al. (1988) for details on the functional analytic setting and terminology:

A) A generates a strongly continuous $\left(C_{0}\right)$ semigroup $T(t)$ on $\mathcal{X}$

B) $(\mathbf{A}, \mathbf{B})$ is exponentially stabilizable, i.e., there exists $\mathbf{F}: \operatorname{dom}(\mathbf{A}) \mapsto \mathcal{U}$ such that $\mathbf{A}+\mathbf{B F}$ generates an exponentially stable $C_{0}$-semigroup $\mathbf{S}(\mathbf{t})$; in case this is true for $\mathbf{F}=0$, we call $\mathbf{A}$ (exponentially) stable.

C) $(\mathbf{A}, \mathbf{C})$ is exponentially detectable, i.e., $\left(\mathbf{A}^{*}, \mathbf{C}^{*}\right)$ is exponentially stabilizable;

D) $\mathbf{B}, \mathbf{C}$ are finite-rank and bounded.

Under the given assumptions, the system $\Sigma(\mathbf{A}, \mathbf{B}, \mathbf{C})$ has a transfer function

$$
\mathbf{G}=\mathbf{C}(s \mathbf{I}-\mathbf{A})^{-1} \mathbf{B} \in L_{\infty},
$$

which is the Laplace transform of $\mathbf{h}(t):=\mathbf{C T}(t) \mathbf{B}$ and symbol of the Hankel operator

$$
\begin{aligned}
\mathbf{H} & : L_{2}(0, \infty ; \mathbf{U}) \mapsto L_{2}(0, \infty ; \mathbf{Y}), \\
(\mathbf{H u})(t) & :=\int_{0}^{\infty} \mathbf{h}(t+\tau) \mathbf{u}(\tau) d \tau .
\end{aligned}
$$

If, in addition, $\mathbf{A}$ is exponentially stable, $\mathbf{G}$ is in the Hardy space $H_{\infty}$. In this case, $\mathbf{H}$ is compact with countable many singular values $\sigma_{j}, j=1, \ldots, \infty$, called the Hankel singular values (HSVs) of G. Moreover, $\sum_{j=1}^{\infty} \sigma_{j}<\infty$. The 2induced operator norm is the $H_{\infty}$ norm; here,

$$
\|\mathbf{G}\|_{H_{\infty}}=\sum_{j=1}^{\infty} \sigma_{j}
$$

Remark 2.1. All results in the following can also be proved using the weaker assumption that $\Sigma(\mathbf{A}, \mathbf{B}, \mathbf{C})$ is a Pritchard-Salomon system (Curtain (2003)), allowing for certain unboundedness of $\mathbf{B}, \mathbf{C}$, which is required for boundary control problems. An even more general setting is considered in Guiver and Opmeer (2012), where it is only assumed that the Hankel operator related to $\Sigma(\mathbf{A}, \mathbf{B}, \mathbf{C})$ is nuclear. This includes a much larger class of boundary control problems. We conjecture that all results in this paper remain true in this setting, though only the BT case is considered in Guiver and Opmeer (2012).

BT is then based on the following results, where due to space limitations, we have to leave out some of the functional analytic details to make all the given statements 
precise, see Glover et al. (1988) and Curtain (2003) for a full account of the theory.

Definition 2.2. (Glover et al. (1988)). For a transfer function $\mathbf{G} \in H_{\infty}, \Sigma(\mathbf{A}, \mathbf{B}, \mathbf{C})$ is a balanced realization of $\mathbf{G}$ if the controllability and observability Gramians, given by the unique self-adjoint positive semidefinite solutions of the Lyapunov equations

$$
\begin{aligned}
\mathbf{A P z}+\mathbf{P A}^{*} \mathbf{z}+\mathbf{B B}^{*} \mathbf{z}=0 & \forall \mathbf{z} \in \operatorname{dom}\left(\mathbf{A}^{*}\right) \\
\mathbf{A}^{*} \mathbf{Q z}+\mathbf{Q A z} \mathbf{\mathbf { z }}+\mathbf{C}^{*} \mathbf{C} \mathbf{z}=0 & \forall \mathbf{z} \in \operatorname{dom}(\mathbf{A})
\end{aligned}
$$

are diagonal operators $\mathbf{P}=\mathbf{Q}=\operatorname{diag}\left(\sigma_{j}\right)=: \boldsymbol{\Sigma}$.

Theorem 2.3. (Glover et al. (1988)). For $\mathbf{G} \in H_{\infty}$ with balanced realization $\Sigma(\mathbf{A}, \mathbf{B}, \mathbf{C})$ and $\mathbf{P}=\mathbf{Q}=\operatorname{diag}\left(\sigma_{j}\right)$, choose $r$ with $\sigma_{r}>\sigma_{r+1}$ and partition $\Sigma(\mathbf{A}, \mathbf{B}, \mathbf{C})$ according to

so that

$$
\mathbf{P}_{r}=\mathbf{Q}_{r}=\operatorname{diag}\left(\sigma_{1}, \ldots, \sigma_{r}\right),
$$

$$
\mathbf{A}=\left[\begin{array}{cc}
\mathbf{A}_{r} & * \\
* & *
\end{array}\right], \quad \mathbf{B}=\left[\begin{array}{c}
\mathbf{B}_{r} \\
*
\end{array}\right], \quad \mathbf{C}=\left[\mathbf{C}_{r} *\right],
$$

then the reduced-order model $\Sigma_{r}\left(\mathbf{A}_{r}, \mathbf{B}_{r}, \mathbf{C}_{r}\right)$ is stable with transfer

$$
\left\|\mathbf{G}-\mathbf{G}_{r}\right\|_{H_{\infty}} \leq 2 \sum_{j=r+1}^{\infty} \sigma_{j} .
$$

For systems that are not exponentially stable, BT is not applicable in the form presented above. Among other things, the $H_{\infty}$-norm is not defined for unstable systems. An alternative is provided by LQG balancing:

Definition 2.4. (Curtain (2003)). For a transfer function $\mathbf{G} \in L_{\infty}, \Sigma(\mathbf{A}, \mathbf{B}, \mathbf{C})$ is an $L Q G$-balanced realization of $\mathbf{G}$ if the unique self-adjoint, positive semidefinite, stabilizing solutions of the operator Riccati equations

$$
\mathbf{A P z}+\mathbf{P A}^{*} \mathbf{z}-\mathbf{P C}^{*} \mathbf{C P z}+\mathbf{B B}^{*} \mathbf{z}=0 \quad \text { for } \mathbf{z} \in \operatorname{dom}\left(\mathbf{A}^{*}\right)
$$$$
\mathbf{A}^{*} \mathbf{Q z}+\mathbf{Q A z}-\mathbf{Q B B}^{*} \mathbf{Q z}+\mathbf{C}^{*} \mathbf{C z}=0 \text { for } \mathbf{z} \in \operatorname{dom}(\mathbf{A})
$$

are bounded and diagonal, $\mathbf{P}=\mathbf{Q}=\operatorname{diag}\left(\gamma_{j}\right)=: \mathbf{\Gamma}$. Here, $\mathbf{P}$ is stabilizing if $\mathbf{A}-\mathbf{P} \mathbf{C}^{*} \mathbf{C}$ generates an exponentially stable $C_{0}$-semigroup.

Abstract LQG BT is then derived similar to BT:

Theorem 2.5. (Curtain (2003)). Given G $\in L_{\infty}$ with LQG balanced realization $\Sigma(\mathbf{A}, \mathbf{B}, \mathbf{C})$ and $\mathbf{P}=\mathbf{Q}=$ $\operatorname{diag}\left(\gamma_{j}\right)$, choose $r$ with $\gamma_{r}>\gamma_{r+1}$ and partition $\Sigma(\mathbf{A}, \mathbf{B}, \mathbf{C})$ according to $\mathbf{P}_{r}=\mathbf{Q}_{r}=\operatorname{diag}\left(\gamma_{1}, \ldots, \gamma_{r}\right)$, so that

$$
\mathbf{A}=\left[\begin{array}{cc}
\mathbf{A}_{r} & * \\
* & *
\end{array}\right], \quad \mathbf{B}=\left[\begin{array}{c}
\mathbf{B}_{r} \\
*
\end{array}\right], \quad \mathbf{C}=\left[\begin{array}{l}
\mathbf{C}_{r} *
\end{array}\right],
$$

then the reduced-order model is the LQG balanced system $\Sigma_{r}\left(\mathbf{A}_{r}, \mathbf{B}_{r}, \mathbf{C}_{r}\right)$ with transfer function $\mathbf{G}_{r}$ satisfying function $\mathbf{G}_{r}$ satisfying

$$
\left\|[\mathbf{N}, \mathbf{M}]-\left[\mathbf{N}_{r}, \mathbf{M}_{r}\right]\right\|_{H_{\infty}} \leq 2 \sum_{j=r+1}^{\infty} \frac{\gamma_{j}}{\sqrt{1+\gamma_{j}^{2}}},
$$

where $\mathbf{G}=\mathbf{M}^{-1} \mathbf{N}$ and $\mathbf{G}_{r}=\mathbf{M}_{r}^{-1} \mathbf{N}_{r}$ are normalized left coprime factorizations, i.e., $\mathbf{M}, \mathbf{M}_{r}, \mathbf{N}, \mathbf{N}_{r}$ are all stable transfer functions.

Of course, none of the model reduction methods described above can be implemented in practice. In order to apply this to parabolic control systems. one has to perform a twostep approach: first, use a high fidelity approximation in the spatial variables of (1)-(2), then apply the corresponding finite-dimensional model reduction techniques to the resulting finite-dimensional systems. We will discuss this in the next section.

\section{COMPUTATIONALLY EFFICIENT BT}

\subsection{Computational procedure - a sketch}

In order to apply (LQG) BT to a parabolic control system, we apply a spatial discretization by the finite element, finite volume, or finite differences method (FEM, FVM, FDM) to (1)-(3) and obtain a finite-dimensional system on $\mathcal{X}_{n} \subset \mathcal{X}$ with $\operatorname{dim} \mathcal{X}_{n}=n$ :

$$
\begin{aligned}
& \dot{x}=A x+B u, \quad x(0)=x_{0}, \\
& y=C x,
\end{aligned}
$$

where $A \in \mathbb{R}^{n \times n}, B \in \mathbb{R}^{n \times m}, C \in \mathbb{R}^{p \times n}$, with corresponding algebraic Lyapunov equations

$$
A P+P A^{T}+B B^{T}=0, \quad A^{T} Q+Q A+C^{T} C=0,
$$

and algebraic Riccati equations (AREs)

$$
\begin{aligned}
& 0=A P+P A^{T}-P C^{T} C P+B B^{T} \\
& 0=A^{T} Q+Q A-Q B B^{T} Q+C^{T} C .
\end{aligned}
$$

Here, we have ignored the possible occurrence of a mass matrix $M$ which can be treated in several ways, see Benner (2004). For now, we assume that $A, B$ also represent $M^{-1} A, M^{-1} B$, where, of course, this matrix is never formed explicitly in numerical computations. Dealing with $M$ is merely a matter of implementation and using the right scalar product in the numerical algorithms, and we refrain from presenting these technical matters here.

So the main computational challenge consists in solving (11) or (12) efficiently. Note that it suffices to compute low-rank factors of $P, Q$ as only these are needed in the SR implmentation of (LQG) BT: given $S \in \mathbb{R}^{n_{P} \times n}, R \in$ $\mathbb{R}^{n_{Q} \times n}, n_{P}, n_{Q} \ll n$ so that $P=S^{T} S, Q=R^{T} R$, compute the singular value decmposition (SVD)

$$
S R^{T}=\left[U_{1}, U_{2}\right]\left[\begin{array}{cc}
\Sigma_{1} & \\
& \Sigma_{2}
\end{array}\right]\left[\begin{array}{c}
V_{1}^{T} \\
V_{2}^{T}
\end{array}\right]
$$

and set $W=R^{T} V_{1} \Sigma_{1}^{-1 / 2}, V=S^{T} U_{1} \Sigma_{1}^{-1 / 2}$. Then the (LQG) BT reduced-order model is $(\hat{A}, \hat{B}, \hat{C})=$ $\left(W^{T} A V, W^{T} B, C V\right)$.

In practice, the existence of low-rank factors of $P, Q$ cannot be assumed, but in many situations, very good low-rank approximations to $P, Q$ of the above form can be expected as $P, Q$ are finite-dimensional approximations to the solutions of the operator Lyapunov and Riccati equations (7) and (8), respectively, and these solution operators are nuclear or at least compact in many situations. So for all practical purposes, we compute low-rank approximations to the system Gramians (solutions of (11)) or LQG Gramians (solutions of (12)). There are now many methods to perform this computation even for high fidelity models with $n=10^{6}$ or more, see, e.g., Benner (2004, 2009); Benner et al. (2008); Morris and Navasca (2005) and references therein.

Remark 3.1. As a by-product of LQG BT, one can obtain a reduced-order LQR or LQG controller, using the solutions $\hat{P}, \hat{Q}$ of the reduced-order AREs corresponding to $(\hat{A}, \hat{B}, \hat{C})$, which are by-products of some computational schemes to solve large-scale AREs. The LQR (state feedback) controller later used in Example 4.3 is given by 
$\hat{B}^{T} \hat{Q} V^{T}$, the LQG controller requires both ARE solutions and is more complicated to describe; we omit the details.

\subsection{Output error bound}

In Curtain (2003), it is shown that under rather generic assumptions for the discretization scheme, the solutions of $(11),(12)$ converge to those of $(7),(8)$, and that the reduced-order models thus obtained converge to those obtained in the abstract setting discussed in the previous section. From a practical point of view, it may be more interesting to study the error obtained from the 2-step approach consisting of spatial semi-discretization and finitedimensional (LQG) BT.

Assuming $\mathbf{U}=\mathbb{R}^{m}, \mathbf{Y}=\mathbb{R}^{p}$ as indicated before, $\mathbf{C} \in$ $\mathcal{L}\left(\mathcal{X}, \mathbb{R}^{p}\right)$ bounded, $C=\left.\mathbf{C}\right|_{\mathcal{X}_{n}}, \mathcal{X}_{n} \subset \mathcal{X}$, we obtain the following simple inequalities, separating the discretization from the model reduction error:

$$
\begin{aligned}
\|\mathbf{y}-\hat{y}\|_{L_{2}\left(0, T ; \mathbb{R}^{p}\right)} \\
\leq\|\mathbf{y}-y\|_{L_{2}\left(0, T ; \mathbb{R}^{p}\right)}+\|y-\hat{y}\|_{L_{2}\left(0, T ; \mathbb{R}^{p}\right)} \\
=\|\mathbf{C} \mathbf{x}-C x\|_{L_{2}\left(0, T ; \mathbb{R}^{p}\right)}+\|y-\hat{y}\|_{L_{2}\left(0, T ; \mathbb{R}^{p}\right)} \\
\leq\|\mathbf{C x}-\mathbf{C} x\|_{L_{2}\left(0, T ; \mathbb{R}^{p}\right)}+\underbrace{\|\mathbf{C} x-C x\|_{L_{2}\left(0, T ; \mathbb{R}^{p}\right)}}_{=0} \\
\quad+\|y-\hat{y}\|_{L_{2}\left(0, T ; \mathbb{R}^{p}\right)} \\
\leq \underbrace{\|\mathbf{C}\|}_{=: c} \cdot \underbrace{\|\mathbf{x}-x\|_{L_{2}(0, T ; \mathcal{X})}}_{\text {discret. error }}+\underbrace{\|y-\hat{y}\|_{L_{2}\left(0, T ; \mathbb{R}^{p}\right)}}_{\text {model reduction error }},
\end{aligned}
$$

where $\|\cdot\|$ denotes the induced operator norm in $\mathcal{L}\left(\mathcal{X}, \mathbb{R}^{p}\right)$.

We thus obtain the following error bound/estimate for $\mathrm{BT} / \mathrm{LQG} \mathrm{BT}$, where the LQG case is more difficult to describe than the BT case due to the more complicated structure of the error bound (9) and therefore rather provides an estimate than a bound:

Corollary 3.2. a) For BT, we get

$$
\begin{aligned}
& \|\mathbf{y}-\hat{y}\|_{L_{2}\left(0, T ; \mathbb{R}^{p}\right)} \\
& \leq c\|\mathbf{x}-x\|_{L_{2}(0, T ; \mathcal{X})}+2\|u\|_{L_{2}\left(0, T ; \mathbb{R}^{p}\right)} \sum_{j=r+1}^{n} \sigma_{j} .
\end{aligned}
$$

b) For LQG BT, we have

$$
\begin{aligned}
& \|y-\hat{y}\|_{L_{2}\left(0, T ; \mathbb{R}^{p}\right)} \\
& \lesssim c\|\mathbf{x}-x\|_{L_{2}(0, T ; \mathcal{X})}+2\|u\|_{L_{2}\left(0, T ; \mathbb{R}^{p}\right)} \sum_{j=r+1}^{n} \frac{\gamma_{j}}{\sqrt{1+\gamma_{j}^{2}}} .
\end{aligned}
$$

Now using standard theory for error bounds of semidiscretizations of parabolic problems under usual smoothness assumptions and coercivity of the associated elliptic bilinear form (e.g., Knabner and Angermann (2000), Sect. 6.2), we can conclude that the output error in the $L_{2}$-norm is bounded by an exponentially decaying term depending only on the error in the initial condition, a factor proportional to $h^{2}$ (with $h$ being the meshsize of the spatial discretization), and the model reduction error. Ignoring the error in the initial condition, it is therefore recommended to balance the discretization and model reduction errors. Denoting the right hand sides in (14) and (15) by $\delta(r)$, it is reasonable to choose the order $r$ of the reduced-order model adaptively by solving

$$
\delta(r) \leq \tau \cdot h^{2},
$$

with a user-specified tolerance $\tau$ and $h$ the meshsize of the used spatial grid.

Note that using the computational scheme based on approximate low-rank factors of the Gramians, we do not have all $n$ HSVs at hand and need to estimate $\delta(r)$ using only those provided by the SVD (13).

Remark 3.3. A different approach to tackle the computational issues arising in BT applied to infinite-dimensional systems is proposed in Reis and Wollner (2012). There, the authors define a computational scheme in infinite dimensions that allows, e.g., to use adaptive FEM. This approach is very promising due to its greater flexibility, but needs to be embedded into a FEM package, while the approach described here is easier to use in the sense that it is built upon modules that are readily available in software packages. Nevertheless, the potentials of both directions should be explored and compared in the future.

\section{NUMERICAL EXAMPLES}

In this section, we test the two suggested methods BT and LQG BT for two test examples from the literature. The main aim here is to illustrate that reliable reduced-order models are obtained using BT and LQG BT approaches that allow on the one hand to compute feedback controllers and on the other hand, to reconstruct even the state. Due to space limitations, more sophisticated tests have to be reported elsewhere.

All computations were done using MATLAB ${ }^{\circledR}$ with IEEE double precision arithmetic.

In the first example, we compare the model reduction behavior of BT and LQG BT.

Example 4.1. (Leibfritz (2004), Section 3.2.1). In this example, the full state-space model results from a semidiscretized boundary control problem for 2D heat flow in copper on a rectangular domain. Section 3.2.1. The control acts on two sides via Robin boundary conditions. A finite differences discretizations leads to a system of order $n=4496$, with $m=2$ inputs. Four sensor locations result in $p=4$. Full details are provided in Leibfritz (2004).

The numerical ranks of the computed Gramians are 68 and 124, respectively, for BT, while for LQG BT both have rank 210 . The computed reduced-order model in both cases is of order $r=10$, based on the grid size $h=0.015$, so that we truncate the HSVs as soon as their estimated tail sums up to a number smaller than $h^{2}$ (i.e., $\tau=1$ in (16)). The HSVs for the standard and LQG Gramians are shown in Fig. 1. Fig. 2 shows that both reduced-order models match the transfer function of the original system very well. Clearly, both methods satisfy their estimated error bounds.

In the next example, we demonstrate that despite its focus on approximating the input-output map of a system, BT can also be used to reconstruct the state trajectories. As in other model reduction methods aiming at state approximation like POD, one may use the truncation 


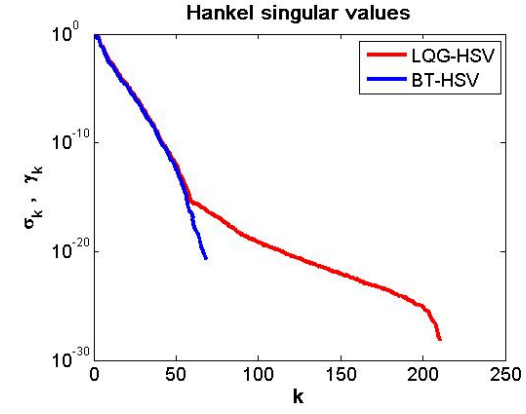

Fig. 1. Example 4.1, decay of (LQG) HSVs.
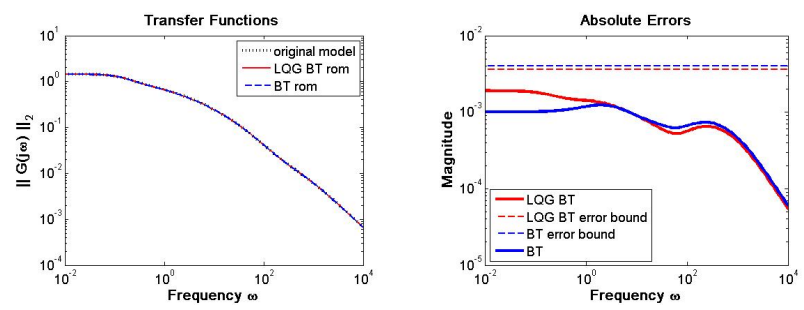

Fig. 2. Example 4.1, original and reduced transfer functions (left), absolute errors (right).

matrices to construct global shape functions. For BT, taking the trial space $\mathcal{V}$ used by BT, one can generate a state approximation by

$$
x(t) \approx V \hat{x}(t)=\sum_{k=1}^{r} v_{k} \hat{x}_{k}(t),
$$

where $V=\left[v_{1}, \ldots, v_{r}\right]$. Thus, the $v_{k}$ are interpreted as discrete global shape functions, analogous to POD modes. Therefore, we call them BT modes.

Example 4.2. Again, we use the heat equation on the unit square as test example. This time, we use point control in a region $\Omega_{u} \subset \Omega$ and homogeneous Dirichlet boundary conditions. We generate the full model as finite differences discretization using the modified LYAPACK Penzl (2000) demo function demo_m1. We employ $h=1 / 64$ and a control domain $\Omega_{u}=[0.2,0.6] \times[0.2,0.25]$ as well as an observation domain $\Omega_{\mathrm{obs}}=[0.2,0.25] \times[0.2,0.25]$.

Using again the adapted criterion (16) with $\tau=1$, we obtain a reduced-order model of dimension $r=6$. The corresponding BT modes are shown in Fig. 3. Like for Fourier modes, the first modes contain low-frequency, global information, while the next BT modes resolve finer scales at higher frequencies.

For comparison purposes, we feed the original $(n=4,096)$ and reduced-order $(r=6)$ system with the control function $u(t)=10 \cos (t)$. The state simulation is performed using a semi-implicit Euler scheme where the state is discretized backward and the control forward in time. Fig. 4 shows the simulated states at $t=1$ for the full and reduced-order models. In the eyeball norm, no difference can be detected.

In our last example, we show the performance of a feedback controller based on the reduced-order model.

Example 4.3. As in the previous example, we use the heat equation on the unit square with homogeneous Dirichlet boundaries, point control and observation with the same
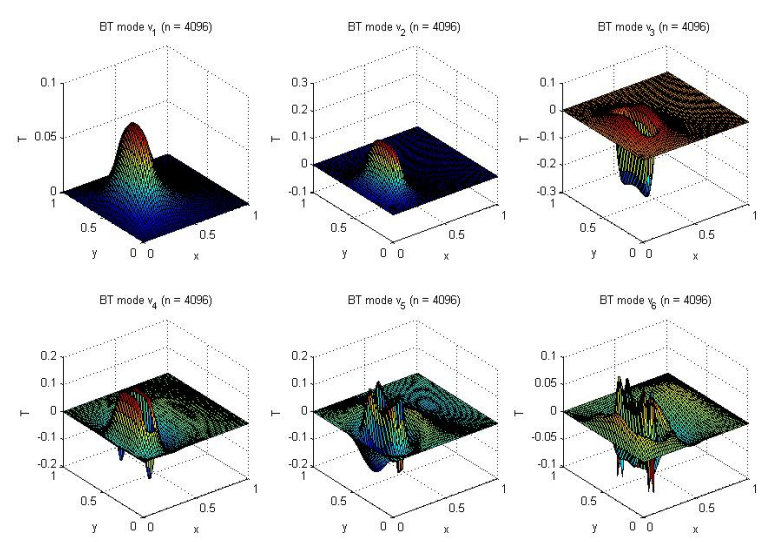

Fig. 3. Example 4.2, computed BT modes $(r=6)$.
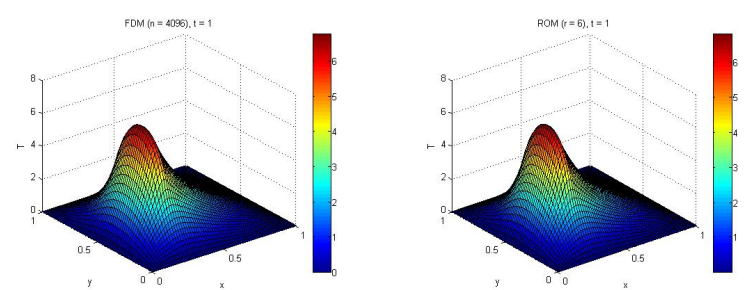

Fig. 4. Example 4.2, simulated states of the original (left) and reduced-order (right) model at $t=1$ using $u(t)=$ $10 \cos (t)$

$\Omega_{u}, \Omega_{\mathrm{obs}}$ as in the previous example. The model is generated using the LYAPACK demo function demo_r1 with $h=\frac{1}{150}$, leading to a full-order model of size $n=22,500$. The computed numerical ranks of the system Gramians are 31 and 26, respectively. Using this time (16) with $\tau=50$, we obtain a BT reduced-order model of size $r=6$. The estimated BT error bound is $\delta=1.7 \cdot 10^{-3}$.

As a simple controller, we solve the LQR problem for the full as well as the reduced-order model to obtain a linear state feedback. The application of the corresponding feedback gains to the full state in the interval $[0,0.2]$ is illustrated in Fig. 6, while the resulting errors are shown in Fig. 5. It should be noted that the relative errors increase towards the end of the interval as controls and outputs approach zero rapidly.

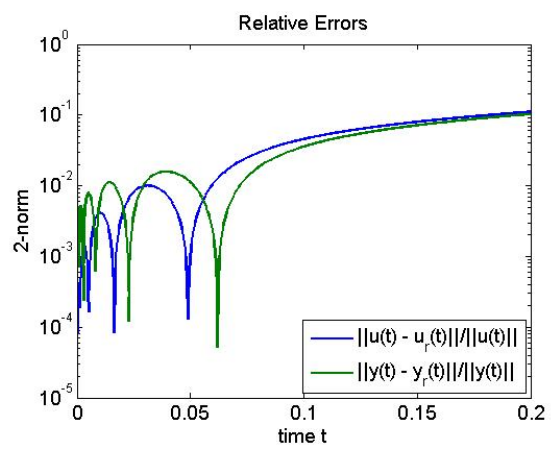

Fig. 5. Example 4.3, relative errors between LQR controls and resulting outputs obtained from full- and reduced-order models. 

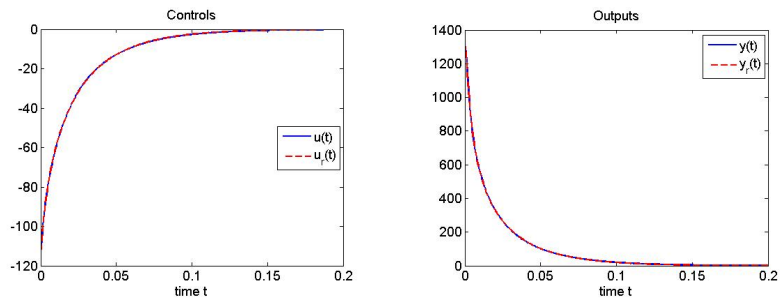

Fig. 6. Example 4.3, simulated states of the original (left) and reduced-order (right) model at $t=1$ using $u(t)=$ $10 \cos (t)$.

\section{CONCLUSIONS AND OUTLOOK}

We have discussed balancing-related model reduction methods for parabolic control systems. Both variants, BT based on balancing the controllability and observabilty Gramians and LQG BT employing the ARE solutions obtained from an LQG design, yield reliable reducedorder models that can be used to approximate the system outputs as well as the states of the system and to compute feedback controllers. Future work will include an automatized full implementation of these model reduction concepts in an open FEM package as well as the derivation of analogous results based on $H_{\infty}$ balancing Mustafa and Glover (1991). Moreover, we also plan to extend our results to parabolic systems containing weak nonlinearities.

\section{REFERENCES}

Antoulas, A. (2005). Approximation of Large-Scale Dynamical Systems. SIAM Publications, Philadelphia, PA.

Banks, H.T. and Kunisch, K. (1984). The linear regulator problem for parabolic systems. SIAM J. Control Optim., 22(5), 684-698.

Benner, P. (2004). Solving large-scale control problems. IEEE Control Systems Magazine, 14(1), 44-59.

Benner, P. (2009). System-theoretic methods for model reduction of large-scale systems: Simulation, control, and inverse problems. In I. Troch and F. Breitenecker (eds.), Proceedings of MathMod 2009, Vienna, February 11-13, 2009, volume 35 of ARGESIM Report, 126-145.

Benner, P., Li, J.R., and Penzl, T. (2008). Numerical solution of large Lyapunov equations, Riccati equations, and linear-quadratic control problems. Numer. Lin. Alg. Appl., 15(9), 755-777.

Benner, P., Mehrmann, V., and Sorensen, D. (eds.) (2005). Dimension Reduction of Large-Scale Systems, volume 45 of Lecture Notes in Computational Science and Engineering. Springer-Verlag, Berlin/Heidelberg, Germany.

Bensoussan, A., Da Prato, G., Delfour, M.C., and Mitter, S.K. (2007). Representation and control of infinite dimensional systems. Systems \& Control: Foundations \& Applications. Birkhäuser Boston Inc., Boston, MA, second edition.

Burns, J.A. and Hulsing, K.P. (2001). Numerical methods for approximating functional gains in LQR boundary control problems. Math. Comput. Modelling, 33(1-3), 89-100.

Curtain, R. and Zwart, H. (1995). An Introduction to Infinite-Dimensional Linear Systems Theory, volume 21 of Texts in Applied Mathematics. Springer-Verlag, NY.

Curtain, R.F. (2003). Model reduction for control design for distributed parameter systems. In Research direc- tions in distributed parameter systems (Raleigh, NC, 2000), volume 27 of Frontiers Appl. Math., 95-121. SIAM, Philadelphia, PA.

Glover, K., Curtain, R.F., and Partington, J.R. (1988). Realisation and approximation of linear infinitedimensional systems with error bounds. SIAM J. Control Optim., 26(4), 863-898.

Guiver, C. and Opmeer, M. (2012). Model reduction by balanced truncation for systems with nuclear Hankel operators. Preprint.

Hinze, M. and Volkwein, S. (2005). Proper orthognonal decomposition surrogate models for nonlinear dynamical systems: Error estimates and suboptimal control. Chapter 10 (pages 261-306) of Benner et al. (2005).

Knabner, P. and Angermann, L. (2000). Numerical Methods for Partial Differential Equations. Springer-Verlag, Berlin.

Kunisch, K. and Volkwein, S. (2001). Galerkin proper orthogonal decomposition methods for parabolic problems. Numer. Math., 90(1), 117-148.

Lasiecka, I. and Triggiani, R. (2000). Control theory for partial differential equations: continuous and approximation theories. I, volume 74 of Encyclopedia of Mathematics and its Applications. Cambridge University Press, Cambridge. Abstract parabolic systems.

Leibfritz, F. (2004). COMPl $\mathrm{COM}_{e}$ : COnstraint Matrixoptimization Problem library - a collection of test examples for nonlinear semidefinite programs, control system design and related problems. Technical report, http://www . complib.de/.

Morris, K.A. (1996). Design of finite-dimensional controllers for infinite-dimensional systems by approximation. J. Math. Systems Estim. Control, 6(2), 151-180.

Morris, K. and Navasca, C. (2005). Solution of algebraic Riccati equations arising in control of partial differential equations. In Control and boundary analysis, volume 240 of Lect. Notes Pure Appl. Math., 257-280. Chapman \& Hall/CRC, Boca Raton, FL.

Mustafa, D. and Glover, K. (1991). Controller reduction by $H_{\infty}$-balanced truncation. IEEE Trans. Automat. Control, 36(6), 668-682.

Obinata, G. and Anderson, B. (2001). Model Reduction for Control System Design. Communications and Control Engineering Series. Springer-Verlag, London, UK.

Penzl, T. (2000). LyapACK Users Guide. Technical Report SFB393/00-33, Sonderforschungsbereich $393 \mathrm{Nu}$ merische Simulation auf massiv parallelen Rechnern, TU Chemnitz, 09107 Chemnitz, FRG. Available from http://www . tu-chemnitz.de/sfb393/sfb00pr.html.

Reis, T. and Wollner, W. (2012). Finite-rank ADI iteration for operator Lyapunov equations. Hamburger Beiträge zur Angewandten Mathematik 2012-09, Universität Hamburg.

Rosen, I. and Wang, C. (1995). A multi-level technique for the approximate solution of operator Lyapunov and algebraic Riccati equations. SIAM J. Numer. Anal., $32(2), 514-541$.

Tröltzsch, F. (2010). Optimal Control of Partial Differential Equations. Theory, Methods and Applications. American Mathematical Society, Providence, RI.

Willcox, K. and Peraire, J. (2002). Balanced model reduction via the proper orthogonal decomposition. AIAA Journal, 40(11), 2323-2330. 